\title{
PROPUESTA DE UN SISTEMA TÉCNICO DE VIVIENDA MÍNIMA MODULAR PREFABRICADA
}

\section{PROPOSAL FOR A PREFABRICATED TECHNICAL SYSTEM TO BUILD MODULAR LOW INCOME HOUSING}

\author{
Byron Andrés Chuñir Bueno ${ }^{1}$, Francisco Adolfo Rodríguez Cedillo ${ }^{1}$, Vladimir \\ Eugenio Carrasco Castro (Tutor) ${ }^{1}$ \\ ${ }^{1}$ Escuela de Ingeniería Civil y Gerencia de Construcciones, Facultad de Ciencia y \\ Tecnología \\ Universidad del Azuay, Cuenca, Ecuador \\ byron95@es.uazuay.edu.ec, franco-369@hotmail.com, vcarrasco@uazuay.edu.ec
}

Recibido: Junio, 2018 Aceptado: Diciembre, 2018

\section{RESUMEN}

Debido al alto requerimiento de vivienda de bajo costo alrededor del planeta, la falta de oferta de parte del sector formal de la construcción, así como la poca inversión en investigación y desarrollo en el sector, han provocado que la construcción de viviendas sea realizada por el sector informal.

Este sector posee conocimientos empíricos y no técnicos sobre la construcción, lo que ocasiona el incumplimiento de las normas de construcción y genera riesgos a la vida de la población frente a fenómenos naturales.

La presente propuesta tiene la finalidad de desarrollar un sistema de construcción de viviendas modulares que garantice el cumplimiento de las normas de construcción, logrando brindar seguridad y generando costos accesibles para la población.

Este sistema se fundamenta en las investigaciones del campo de la Ingeniería Civil y la Gerencia en Construcciones.

Palabras clave: vivienda, mínima, modular, prefabricada, estructuras, programa, comercial.

\section{ABSTRACT}

Due to the high demand for low-cost housing around the planet, the lack of housing supply from the formal construction sector, as well as the little investment in research and development in the area, have led to the construction of homes by the informal sector.

This sector has empirical and non-technical knowledge on their construction processes, which leads to non-compliance with building regulations and generates risks to the life of the population in the face of natural phenomena. 
The purpose of this proposal is to develop a modular housing construction system that guarantees compliance with construction standards, providing security and generating affordable costs for the population.

This system is based on research in the field of Civil Engineering and Construction Management.

Keywords: Minimum, housing, modular, prefabricated, structures, commercial, program.

\section{INTRODUCCIÓN}

Debido a la difícil situación económica actual del Ecuador, menos de la mitad de la población del país posee vivienda propia o totalmente pagada. Además, el continuo crecimiento poblacional que según datos del INEN incrementará un $62 \%$ aproximadamente para el año 2050, ocasiona que se genere una gran demanda de viviendas a satisfacer. Esta demanda actualmente está destinada al sector informal de la construcción. El método de construcción empleado por este sector crea riesgos a la vida de la población del país frente a fenómenos naturales, debido a su falta de conocimiento técnico e incumplimiento de las normas establecidas en el país en el ámbito de la construcción.

A causa de esta necesidad surge el desarrollo de sistemas que cuentan con nuevos materiales y técnicas constructivas, las cuales deben garantizar la innovación, seguridad y accesibilidad económica para la población del país.

Estos sistemas deben estar fundamentados en las investigaciones en el campo de la Ingeniería Civil y la Gerencia en Construcciones.

\section{DESARROLLO}

Con el afán de desarrollar un sistema constructivo prefabricado eficiente y de calidad enfocado a la vivienda mínima para la zona de la sierra sur del Ecuador, se optó por elegir el sistema de vivienda modular prefabricada o denominado como construcción modular industrializada puesto que la fabricación del módulo sigue una línea de producción hasta posteriormente ser transportada al lugar de emplazamiento.

Este sistema permite la inclusión de nuevos materiales y técnicas constructivas que consiguen ser la respuesta a una demanda cada vez más exigente en aspectos como: precios, seguridad, calidad constructiva, plazos de ejecución, sostenibilidad e impacto ambiental.

Por lo cual se aprovechó las ventajas que brinda este sistema constructivo, las cuales son:

- Óptimas condiciones de trabajo: debido a un ambiente controlado que permite tener un mayor control de la producción ante condiciones laborales y climáticas. El acceso a herramientas se facilita, se necesita menos entregas de materiales en tiempo, la supervisión del trabajo mejora, de modo que, se logra un mejor control de calidad. 
- Reduce el impacto ambiental: disminuyen los desperdicios de materiales y energía. Se reduce la contaminación del agua, polvo y ruido.

- Planificación estratégica de actividades: con trabajo mejor programado que permite reducir los conflictos entre los operarios de la construcción.

- Control de calidad: los materiales que son usados en este tipo de construcción son de mejor calidad al momento de ser ensamblados, así como en su almacenamiento. Inclusive se reducen las perdidas, desperdicios y espacios de almacenamiento de los mismos.

- La seguridad laboral: se mejoran significativamente las condiciones de trabajo para el personal obrero, debido a que no está expuesto a la intemperie, climas extremos o actividades peligrosas.

- Reducción en los tiempos de ejecución: la producción en fábrica nos brinda la posibilidad de realizar actividades en paralelo (cambio en la secuencia de construcción), es decir se reduce el costo debido a que existe un ahorro en tiempo y mejora del flujo de trabajo (Modular Building Institute, 2018).

Por consiguiente, se emplea el sistema modular prefabricado para la propuesta de la vivienda mínima o de interés social, la misma que está destinada a los grupos de atención prioritaria y a la población en situación de pobreza o vulnerabilidad (Asamblea Nacional, 2016).

Según el Ministerio de Desarrollo Urbano y Vivienda por medio del acuerdo ministerial №220 la propuesta de la vivienda mínima modular está en la clasificación de vivienda tipo 2, la misma que cuenta con un área de $44.25 \mathrm{~m}^{2}$, un costo directo total de $\$ 15,153.55$ dólares americanos y destina para la familia tipo de Ecuador conformada por cuatro integrantes.

Indudablemente, la vivienda mínima satisfacerá las necesidades de sus habitantes en términos: físicos, psicológicos, sociales y ambientales; por medio del cumplimiento de las siguientes características: privacidad, espacios adecuados, accesibilidad física, seguridad, estabilidad y durabilidad estructural, iluminación, calefacción y/o ventilación e incluir infraestructura de servicios básicos. (Arias \& Malo, 2013).

Mediante la consulta bibliográfica se procedió a realizar un análisis arquitectónico con el fin de obtener las dimensiones óptimas y confortables para los espacios interiores de la vivienda (Arredondo Zambrano \& Reyes Bernal, 2013). Se obtiene como resultado la distribución del área de la vivienda en espacios destinados a: dormitorio principal, dormitorio secundario, cocina, sala, comedor, baño general y lavandería; los mismos que se describen en la Tabla 1 y Figura 1:

Tabla 1 Dimensionamiento de las áreas de la vivienda mínima modular.

\begin{tabular}{|l|c|}
\hline \multicolumn{1}{|c|}{ Tipo de habitación } & Área $\left(\mathrm{m}^{2}\right)$ \\
\hline Dormitorio principal & 7.29 \\
\hline Dormitorio secundario & 6.88 \\
\hline Cocina & 7.81 \\
\hline Sala y comedor & 14.75 \\
\hline Baño general & 2.89 \\
\hline
\end{tabular}


Fuente: Autor.

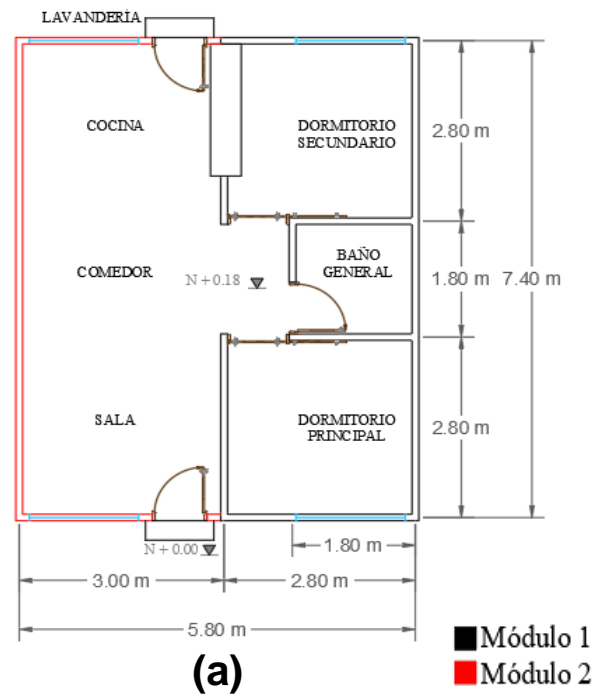

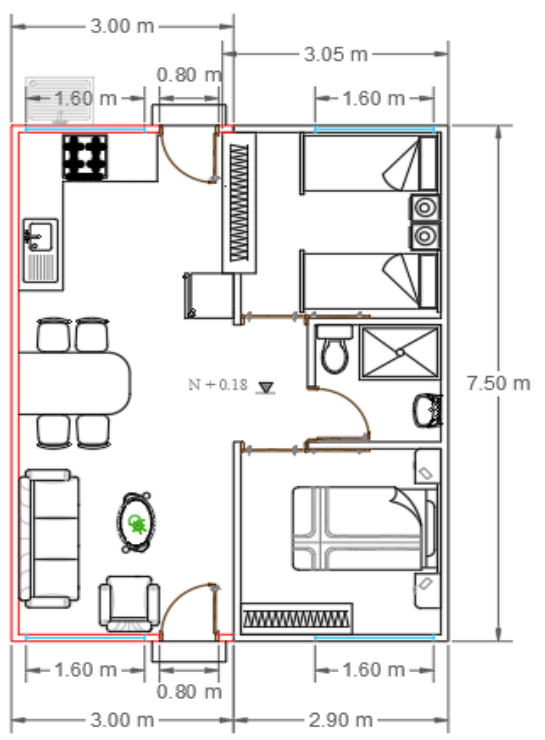

(b)

Figura 1 (a) Distribución de áreas. (b) Distribución de mobiliario de la vivienda mínima modular planta baja.

Fuente: Autor.

Para determinar el sistema estructural óptimo se realizó un análisis a tres diferentes tipos de sistemas: sistema semi-prefabricado con paneles de hormigón, sistema steel framing, sistema metálico con paneles de pared y losa de entrepiso de micro hormigón. Este análisis se basa el en método cualitativo por puntos, el mismo que tiene como objetivo elegir el sistema que presente resultados superiores en los factores considerados como relevantes para el investigador; los mismos que se mencionan a continuación: económico, condiciones de servicio, durabilidad, tiempo de construcción, mantenimiento y reparación.

Al finalizar este análisis se concluyó que los materiales de provisión nacional que presentan las adecuadas características técnicas para la aplicación del sistema de vivienda modular prefabricada es el sistema de estructura metálica con paneles tipo pared y losa de entrepiso de micro hormigón.

A su vez, este sistema proporciona importantes ventajas tales como:

- Aislamiento térmico ya que utiliza poli estireno expandido en sus paneles lo cual mantiene las temperaturas confortables y estables.

- Aísla el sonido ya que contiene un material que absorbe frecuencias altas, medias y bajas, proporcionando un ambiente sin molestias.

- Los paneles al contar con un reforzamiento similar al hormigón armado no podrán ser destruidas en caso de vandalismo, robo o incendios.

- $\quad$ Permite reducir el área y el peso producto de la tabiquería, la misma que no se pudre y es liviana debido a su alma de poli estireno. 
- $\quad$ Crea un sistema alternativo de construcción fácil con menor tiempo de ejecución y mano de obra.

Con respecto al diseño estructural de la vivienda mínima modular se realiza según los parámetros establecidos en la Norma Ecuatoriana de la Construcción para el sistema tipo pórtico, el que se define como un sistema estructural compuesto por un pórtico espacial, sin diagonales, que resiste todas las cargas verticales y horizontales (Ministerio de Ambiente Vivienda y Desarrollo Territorial, 2010), por lo cual, el diseño fue realizado de la siguiente manera:

1. Elección de cargas permanentes y variables (sismo, viento, sobrecarga de uso y granizo) que actúan sobre la estructura en los escenarios de: transporte desde la fábrica al lugar de emplazamiento y la vivienda emplazada en el sitio con la ampliación de la planta alta.

2. Verificar las distintas combinaciones de carga para el escenario más desfavorable para la vivienda, obteniendo como resultado el escenario de la vivienda emplazada en el sitio con la ampliación de la planta alta.

3. Comprobar las secciones elegidas y unión tipo soldadura para los elementos estructurales (columna, vigas y viguetas) referidos a la normativa y especificaciones del AISI., instituto encargado de la regularización de perfilería laminada en frío.

4. Comprobación de derivas máximas permitidas por las NEC producto de la acción de las distintas cargas que se aplican a la estructura, cumpliendo en todos los ejes de la estructura y garantizando la seguridad de la vivienda.

Posteriormente de haber realizado el diseño estructural se procede a la descripción y resultados de la comprobación de los elementos estructurales de la vivienda mínima modular:

- Para columnas se utiliza el perfil metálico rectangular tipo estructural de dimensiones $150 \mathrm{~mm} \times 100 \mathrm{~mm} \times 4 \mathrm{~mm}$ que cumple con la normativa y especificaciones establecidas por el AISI.

- Para vigas se utiliza el perfil metálico rectangular tipo estructural de dimensiones $150 \mathrm{~mm}$ x $100 \mathrm{~mm}$ x $3 \mathrm{~mm}$ que cumple con la normativa y especificaciones establecidas por el AISI.

- Para viguetas se utiliza el perfil metálico rectangular tipo estructural de dimensiones $100 \mathrm{~mm} \times 100 \mathrm{~mm} \times 3 \mathrm{~mm}$ que cumple con la normativa y especificaciones establecidas por el AISI.

- Para la conexión entre los módulos de la vivienda se realizará un cordón de soldadura aplicado en cabeza y pie de columna distribuida en cordones de $40.20 \mathrm{~cm}$ en las dos caras del elemento estructural tipo columna.

- Para conexión entre los elementos columna-viga se realizará un cordón de soldadura por todo el contorno de la sección del elemento viga.

- Para conexión entre los elementos viga-vigueta se realizará un cordón de soldadura por todo el contorno de la sección del elemento vigueta.

Del mismo modo se describe los tipos de paneles a utilizarse en la vivienda mínima modular: 
- Para mampostería vertical se utiliza el panel tipo simple conformado por dos láminas externas de micro hormigón vibro prensado de $1.20 \mathrm{~cm}$ de espesor cada una, una lámina central de poliestireno expandido con espesor de $5 \mathrm{~cm}$ y malla hexagonal en cada cara de la capa de poliestireno. Según la empresa Hormypol este tipo de panel cumple con requerimientos de seguridad y resistencia.

- Para losa de entrepiso utiliza el panel tipo reforzado conformado por dos láminas externas de micro hormigón vibro prensado de $1.20 \mathrm{~cm}$ de espesor cada una, una lámina central de poliestireno expandido con espesor de $5 \mathrm{~cm}$, malla hexagonal de diámetro de $0.5 \mathrm{~mm}$ en cada cara de la capa de poliestireno y malla electrosoldada de diámetro de 5.5 $\mathrm{mm}$ en la cara inferior de la lámina de poliestireno. Según la empresa Hormypol este tipo de panel cumple con requerimientos de seguridad y resistencia.

Con el fin de garantizar los costos mínimos en el proceso de fabricación se plantea una producción esbelta que alcance una productividad eficiente y efectiva, con estrecha orientación al cliente, innovación continua, fuerte concentración sobre técnicas que agregen valor a los procesos y una permanente gestión operativa integrada en el aseguramiento de la calidad (Rivas, 2007).

Adicionalmente, para la compra de los diferentes materiales necesarios para la construcción de la vivienda mínima modular se propone una negociación directa con los proveedores, en la cual se defina una compra continua que garantiza la adquisición de un gran volumen de material en un prolongado periodo de tiempo. Cabe mencionar que los materiales adquiridos deberán ser utilizados de inmediato, lo que evitará la acumulación de inventarios y por ende disminuirá los costos de producción. El propósito de este tipo de compra será de obtener las siguientes ventajas:

- Disminución en los costos de adquisición de los productos por medio de mayores beneficios y descuentos por parte de los proveedores.

- Disminuir los costes de procesos.

- Reducción en las inversiones de capital.

- Mejor gestión de stocks (Begoña González, 2013).

Para garantizar la efectividad del sistema de compra propuesto se debe implementar en los procesos internos de la industria las siguientes características:

- Crear una política y procedimientos de compra uniforme.

- Obtener una calidad uniforme de los materiales adquiridos mediante una estandarización rápida de los productos y servicios.

- Facilitar la planificación de la producción y permitir un mayor control de los pedidos.

Con el objetivo de presentar el correspondiente presupuesto de la vivienda mínima modular se procedió a realizar un análisis de precios unitarios, en el que se considera rendimientos superiores a la construcción tradicional, puesto que se obtienen procesos más eficientes, eficaces en fábrica y se evita la exposición a los agentes atmosféricos que afectan a las obras civiles.

Para la realización del análisis de precios unitarios se considera solamente los costos directos provocados por efecto de la construcción de la parte 
estructural, divisiones de espacios, acabados, transporte y montaje de la misma al lugar de emplazamiento dentro del área urbana de la ciudad de Cuenca; excluyendo los costos indirectos, para el cual se recomienda realizar un estudio detallado con el fin de obtener el porcentaje real del mismo.

A continuación, se procede a presentar los resultados del análisis de precios unitarios en la Tabla 2, Tabla 3 y Tabla 4:

Tabla 2 Presupuesto de producción obra gris de la vivienda mínima modular.

\begin{tabular}{|c|c|c|c|c|c|c|c|}
\hline \multicolumn{8}{|c|}{ Presupuesto conceptual } \\
\hline Ítem & Código & Descripción & Unidad & Cantidad & P.Unitario & & P.Total \\
\hline 1.001 & A 1 & Panel tipo pared para mampostería vertical & $\mathrm{m}^{2}$ & 55 & 11.63 & $\$$ & 639.65 \\
\hline 1.002 & A 2 & Panel losa para entrepiso & $\mathrm{m}^{2}$ & 95.91 & 16.57 & $\$$ & $1,589.23$ \\
\hline 1.003 & A 3 & Perfil metálico tipo columna $(150 \times 100 \times 4 \mathrm{~mm})$ & $\mathrm{kg}$ & 594.80 & 1.67 & $\$$ & 994.51 \\
\hline 1.004 & A 4 & Perfil metálico tipo viga $(150 \times 100 \times 3 \mathrm{~mm})$ & $\mathrm{kg}$ & 1567.57 & 1.67 & $\$$ & $2,620.98$ \\
\hline 1.005 & A 5 & Perfil metálico tipo vigueta $(100 \times 100 \times 3 \mathrm{~mm})$ & $\mathrm{kg}$ & 758.02 & 1.67 & $\$$ & $1,267.41$ \\
\hline & & Costo directo total de & Iucción & & & $\$$ & $7,111.77$ \\
\hline
\end{tabular}

Fuente: Autor.

Tabla 3 Presupuesto conceptual de transporte y montaje de la vivienda mínima modular.

\begin{tabular}{|c|c|c|c|c|c|c|c|}
\hline \multicolumn{8}{|c|}{ Presupuesto conceptual } \\
\hline Ítem & Código & Descripción & Unidad & Cantidad & P.Unitario & \multicolumn{2}{|r|}{ P.Total } \\
\hline 1.006 & $\mathrm{~T} 1$ & Transporte de módulo $2.80 \mathrm{~m}$ & Ton & 6.74 & 9.39 & $\$$ & 63.29 \\
\hline 1.007 & $\mathrm{~T} 2$ & Transporte de módulo $3.00 \mathrm{~m}$ & Ton & 6.74 & 8.58 & $\$$ & 57.83 \\
\hline 1.008 & M1 & Montaje de módulo 2.80 m & Ton & 5.73 & 156.94 & $\$$ & 899.27 \\
\hline 1.009 & M2 & Montaje de módulo 3.00 m & Ton & 5.73 & 152.92 & $\$$ & 876.23 \\
\hline & & \multicolumn{4}{|c|}{ Costo total de transporte y montaje } & $\$$ & $1,896.62$ \\
\hline
\end{tabular}

Fuente: Autor.

Tabla 4 Presupuesto conceptual de acabados de la vivienda mínima modular. 


\begin{tabular}{|c|c|c|c|c|c|c|c|}
\hline \multicolumn{8}{|c|}{ Presupuesto conceptual } \\
\hline Ítem & Código & Descripción & Unidad & Cantidad & P.Unitario & & Total \\
\hline 1.010 & A 10 & Cerámica de piso & $\mathrm{m}^{2}$ & 10.84 & 15.01 & $\$$ & 162.71 \\
\hline 1.011 & A 11 & Cerámica de pared y mesón cocina & $\mathrm{m}^{2}$ & 14.89 & 13.51 & $\$$ & 201.16 \\
\hline 1.012 & A 12 & Piso flotante & $\mathrm{m}^{2}$ & 29.20 & 14.83 & $\$$ & 433.01 \\
\hline 1.013 & A 13 & $\begin{array}{l}\text { Impermeabilización cubierta lámina asfáltica } 3 \\
\text { mm }\end{array}$ & $\mathrm{m}^{2}$ & 47.79 & 11.28 & $\$$ & 539.17 \\
\hline 1.014 & A 14 & Pintura paredes exteriores & $\mathrm{m}^{2}$ & 55.96 & 4.44 & $\$$ & 248.71 \\
\hline 1.015 & A 15 & Pintura paredes interior & $\mathrm{m}^{2}$ & 80.66 & 3.24 & $\$$ & 261.34 \\
\hline 1.016 & A 16 & Accesorios sanitarios y cocina & Unidad & 1.00 & 447.50 & $\$$ & 447.50 \\
\hline 1.017 & A 17 & Puerta principal de madera & $\mathrm{m}^{2}$ & 3.57 & 162.76 & $\$$ & 581.05 \\
\hline 1.018 & A 18 & Puerta corrediza de dormitorio & $\mathrm{m}^{2}$ & 3.57 & 65.09 & $\$$ & 232.37 \\
\hline 1.019 & A 19 & Puerta de madera para baño & $\mathrm{m}^{2}$ & 1.79 & 119.39 & $\$$ & 213.71 \\
\hline 1.020 & A 20 & Rastreras de madera & $\mathrm{ml}$ & 38.29 & 5.89 & $\$$ & 225.49 \\
\hline 1.021 & A 21 & Closet madera & $\mathrm{m}^{2}$ & 2.35 & 112.29 & $\$$ & 263.88 \\
\hline 1.022 & A 22 & Ventanas aluminio y vidrio $4 \mathrm{~mm}$ & $\mathrm{~m}^{2}$ & 7.68 & 68.85 & $\$$ & 528.74 \\
\hline 1.023 & A 23 & $\begin{array}{l}\text { Conexión domiciliaria A.P. 1/2" incluye caja de } \\
\text { medidor }\end{array}$ & Unidad & 1.00 & 121.97 & $\$$ & 121.97 \\
\hline 1.024 & A 24 & Punto agua potable fría & Unidad & 5.00 & 21.06 & $\$$ & 105.32 \\
\hline 1.025 & A 25 & Punto agua servida & Unidad & 7.00 & 23.62 & $\$$ & 165.36 \\
\hline 1.026 & A 26 & Bajante agua lluvia PVC $110 \mathrm{~mm}$ & $\mathrm{~m}$ & 10.00 & 10.27 & $\$$ & 102.73 \\
\hline 1.027 & A 27 & Tablero y caja medidor eléctrico & Unidad & 1.00 & 170.22 & $\$$ & 170.22 \\
\hline 1.028 & A 28 & Acometida telefónica & $\mathrm{m}$ & 7.00 & 2.77 & $\$$ & 19.38 \\
\hline 1.029 & A 29 & Punto luz & Unidad & 8.00 & 33.50 & $\$$ & 267.98 \\
\hline 1.030 & A 30 & Punto tomacorriente $110 \mathrm{~V}$ & Unidad & 13.00 & 33.46 & $\$$ & 434.98 \\
\hline 1.031 & A 31 & Breakers de 1 polo de 40 AMP & Unidad & 1.00 & 8.69 & $\$$ & 8.69 \\
\hline 1.032 & A 32 & Cielo raso gypsum normal & $\mathrm{m}^{2}$ & 40.04 & 10.23 & $\$$ & 409.69 \\
\hline
\end{tabular}

Fuente: Autor.

En la Figura 2, Figura 3 y Figura 4 con la ayuda de unos modelos computarizados, se puede apreciar como luciría la vivienda mínima modular prefabricada terminada.

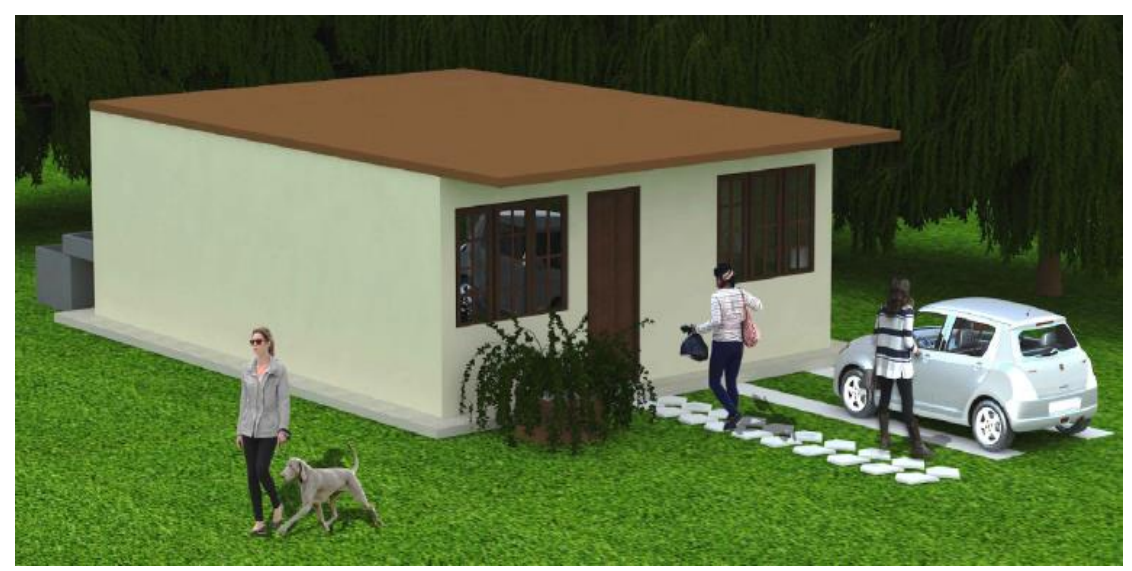

Figura 2 Vista exterior de la vivienda mínima modular. Fuente: Autor. 


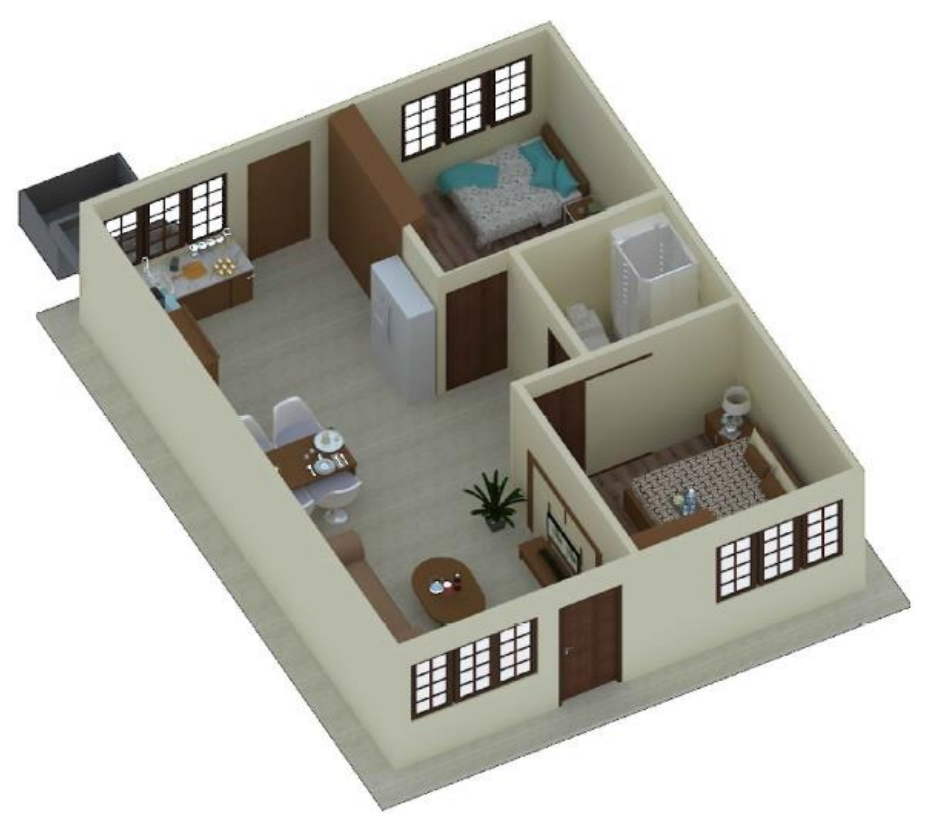

Figura 3 Vista interior de la vivienda mínima modular. Fuente: Autor.

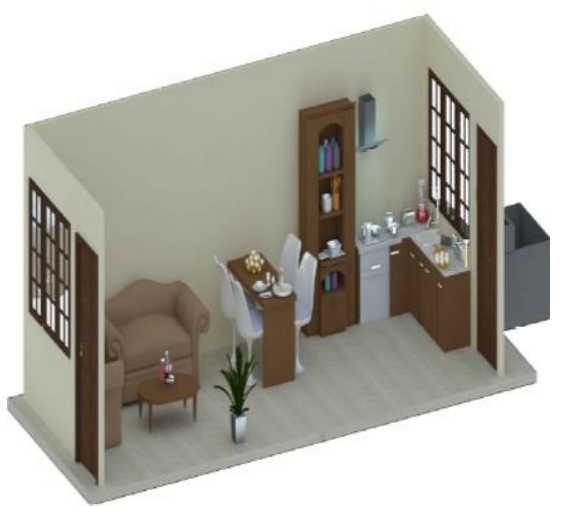

(a)

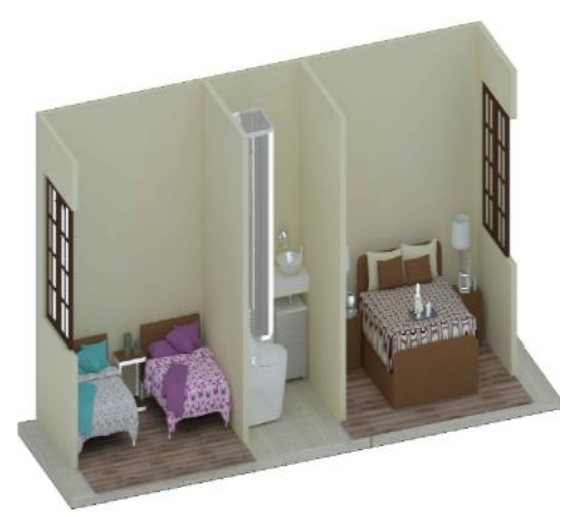

(b)

Figura 4 (a) Vista interior en corte del módulo 1 de la vivienda mínima modular (b) Vista interior en corte del módulo 2 de la vivienda mínima modular.

Fuente: Autor.

Cabe recalcar que en la propuesta de la vivienda mínima modular no se consideran los siguientes rubros:

- Replanteo y nivelación del terreno.

- Movimiento de tierra (corte y relleno del suelo).

- Cimentación de la vivienda.

- Conexión a matrices de las acometidas de servicio básicos (agua potable, alcantarillado, tendido eléctrico y telefónico). 
- Mobiliario interno (juego de sala, comedor y dormitorio).

- Adquisición del terreno.

- Cerramiento de la vivienda.

El costo del terreno es un factor importante a tener en cuenta al momento de referirnos al costo total de la vivienda debido a que este costo se puede incrementar significativamente. En la ciudad de Cuenca el costo de los terrenos es muy alto en comparación con el resto país, por lo que se deberá coordinar con los Gobiernos Autónomos Descentralizados (GADs) la gestión del suelo urbano requerido para los programas de vivienda de interés social conforme con sus respectivas planificaciones. Además, el costo del suelo deberá estar regido a la valoración catastral, el cual refleja el valor real de los inmuebles descontando los valores producto de la distorsión del mercado y siendo competencia de los GADs elaborar normas que contemplen y favorezcan este tipo de producción para facilitar el acceso a: suelo, financiamiento, crédito y asistencia técnica (Ministerio de Desarrollo Urbano y de Vivienda, 2011).

Cabe destacar que en el presente trabajo no se contempla el emplazamiento de la vivienda en un terreno específico, pero en la planificación de la misma se pretende aprovechar de manera eficiente los espacios, por lo cual se plantea un adosamiento lateral doble para las viviendas (una vivienda junto a otra).

Por lo tanto, la vivienda posee una mayor dimensión de fondo de $12.10 \mathrm{~m}$ que de frente de $5.90 \mathrm{~m}$, lo que provoca una reducción en la construcción de infraestructura de servicios básicos (Matrices de agua potable, alcantarillado, tendido eléctrico, pavimentación de veredas y calles, etc.) generando una disminución en el costo del terreno.

\section{ANÁLISIS DE RESULTADOS}

En síntesis, podemos decir que el costo total de la vivienda mínima modular con una superficie de $44.25 \mathrm{~m}^{2}$ es de $\$ 15,153.55$ dólares americanos. Este costo no incluye el costo indirecto. Se debe tener en cuenta que este tipo de propuestas son destinadas a organizaciones de economía popular y solidaria o grupos de población organizada sin fines de lucro. El costo presentado es inferior al rango establecido ( $\$ 20,000.00$ a $\$ 25,000.00$ para una superficie de 42.01 a $54 \mathrm{~m}^{2}$ respectivamente) por el Ministerio de Desarrollo Urbano y de Vivienda (MDUV) del año 2011, incluso si se aumenta los rubros no cuantificados en este estudio e infimamente superior al determinado por los lineamientos mínimos para registro y validación de tipologías de vivienda del programa "casa para todos" del MDUV, con un valor máximo de $\$ 12,500.00$ para una superficie de $49 \mathrm{~m}^{2}$ (Ministerio de Desarrollo Urbano y de Vivienda, 2018).

Por último, se debe tener en consideración que la vivienda esta diseñada para una futura ampliación del área útil mediante la construcción de la planta alta, en la misma que se garantiza la seguridad de quien la habita ante las cargas permanentes y variables que se produzca por el crecimiento de la vivienda. 


\section{CONCLUSIONES}

Mediante el proceso de elección del material se determinó que el sistema de estructura metálica con mampostería vertical y losas de entrepiso de micro hormigón es el más conveniente a utilizar en vivienda mínima modular, los cuales existen en el medio local y presentan mayores beneficios conforme a los criterios de economía, condiciones de servicio, durabilidad, tiempo de construcción, mantenimiento y reparación que estos brindan.

Se estableció las dimensiones óptimas del módulo que brindan mayores ventajas de movilización, colocación en obra y cumplimiento de los criterios arquitectónicos. Además, se normalizó bajos los mismos criterios una posible ampliación de la vivienda mínima modular.

Para el cálculo del diseño estructural mediante la modelación matemática se consideró las cargas y combinaciones que establece la NEC. Como resultado se obtuvo que el escenario más desfavorable a analizar es la que considera la ampliación de la planta alta de la vivienda mínima modular, debido al incremento de cargas que se aplican sobre la estructura base (planta baja).

Al realizar el análisis de los resultados de la modelación se identificó que la combinación de cargas que genera los esfuerzos máximos es la combinación que contempla las siguientes cargas: carga viva, muerta y de granizo. Adicionalmente, se identificó que los elementos estructurales diseñados cumplen ampliamente a los requerimientos que establece el AISI en su normativa. Esto representa una ventaja para la vivienda propuesta frente a otras viviendas de interés social, debido a que la estructura de la vivienda permite una posible ampliación en la primera planta alta de la vivienda mínima modular.

Mediante un análisis de precios unitarios se establece que la vivienda mínima modular presenta un precio accesible para la población con un monto de \$ $15,153.55$ para una vivienda de $44.25 \mathrm{~m}^{2}$ de superficie. Este precio incluye costos de fabricación, transporte e instalación, representando un costo directo de $\$$ 342.45 por $\mathrm{m}^{2}$ de vivienda con acabados, se debe aclarar que no se tomó en cuenta los costos indirectos y los rubros antes mencionados. Finalmente, se puede concluir que la vivienda modular es una opción factible para los programas de vivienda solidaria del país e inclusive para competir dentro del mercado inmobiliario ecuatoriano.

\section{REFERENCIAS}

1. AISI. ESPECIFICACIÓN PARA EL DISEÑO DE MIEMBROS ESTRUCTURALES DE ACERO CONFORMADO EN FRÍO (1996). Estados Unidos.

2. ALQUIMODUL sac. (2015). VIVIENDAS PREFABRICADAS ALQUIMODUL SAC - Construcción modular, módulos prefabricados y contenedores. Retrieved April 24, 2018, from http://www.alquimodulperu.com/noticias/viviendas-prefabricadas/

3. American designe construction. (2018). Casas con estructura de acero steel frame. Retrieved June 11, 2018, from https://casasamericanas.jimdo.com/casas-estructura-de-acero-steel-frame/ 
4. Arciniega Arce, R. S. (2003). Globalización, industriay reestructuración productiva. Convergencia. Revista de Ciencias Sociales (Vol. 10). Universidad Autónoma del Estado de México. Retrieved from http://www.redalyc.org/html/105/10503110/

5. Arias, A. G., \& Malo, D. E. (2013). Coordinación Modular Para La Vivienda Económica Con Elementos Prefabricados. Universidad de Cuenca.

6. Arredondo Zambrano, C. E., \& Reyes Bernal, E. (2013). Manual de vivienda sustentable.pdf. México D.F.: Editorial Trillas S.A.

7. Asamblea Nacional, E. Ley orgánica de ordenamiento territorial, uso y gestión del suelo (2016). Ecuador. Retrieved from http://www.habitatyvivienda.gob.ec/wp-

content/uploads/downloads/2016/08/Ley-Organica-de-Ordenamiento-

Territorial-Uso-y-Gestion-de-Suelo1.pdf

8. Baca Urbina, G. (2001). Evaluacion de Proyectos Gabriel Baca Urbina.pdf. (McGraw-Hill, Ed.) (4ta ed.). México.

9. Begoña González. (2013). Tipos de compras. Ventajas y desventajas. Retrieved May 30, from https://begonagonzalezelejabarrieta.wordpress.com/2013/02/21/tipos-decompras-ventajas-y-desventajas/

10. Bermejo, M., Santos, A. P., Goicolea, J. M., Pérez, A., \& Pérez, A. (2015). Evaluación de acciones explosivas sobre estructuras de hormigón armado mediante elementos finitos. Informes de La Construcción, 67(539), e095. https://doi.org/10.3989/ic.13.121

11. CAPECO. (2003). Costos y presupuestos en edificación. Perú. Retrieved from

https://civilyedaro.files.wordpress.com/2014/08/costos_y_presupuestos_en edificacion_-_capeco_r.pdf

12. Carigliano, S. (2015). ¿Qué es el Análisis Estructural? Retrieved April 23, 2018, from https://skyciv.com/es/education/what-is-structural-analysis/

13. Carpio Rodríguez, E. (2016). Modelo de una vivienda de bajo costo con uso de materiales termoacústico para la población de clase media en Machala. Universidad Técnica de Machala. Retrieved from http://repositorio.utmachala.edu.ec/bitstream/48000/7904/1/TTUAIC_2016 IC_CD0008.pdf

14. Concejo Metropolitano de Quito, Q. Ordenanza 3445 Normas de Arquitectura y Urbanismo (2003). Ecuador. Retrieved from http://www7.quito.gob.ec/mdmq_ordenanzas/Ordenanzas/ORDENANZAS AÑOS ANTERIORES/ORD-3457 - NORMAS DE ARQUITECTURA Y URBANISMO.pdf

15. Del Águila, A. (1996). Sistemas constructivos industrializados. Informes de La Construcción, 48(c), 27-38. https://doi.org/10.3989/ic.1996.v48.i446.983

16. Duarte Martínez, A. S. (2011). Manual Práctico De Control De Costos En Obras Civiles, Aplicado a Construcción De Edificaciones. Universidad Católica Andrés Bello, Venezuela. Retrieved from http://biblioteca2.ucab.edu.ve/anexos/biblioteca/marc/texto/AAS0661.pdf

17. Gutierrez Pulido, H. (2010). Calidad total y productividad. https://doi.org/10.1017/CBO9781107415324.004

18. Hormi2. (2001). MANUAL PRÁCTICO DEL CONSTRUCTOR. Ecuador.

19. Hormypol. (2018). Viviendas Económicas. Retrieved April 23, 2018, from http://hormypol.com/precios-de-construccion-costos-materiales- 
prefabricados-hormigon-quito-guayaquil-cuenca-lojaecuador.php?tablajb=precios_de_construccion $\& p=43 \& t=V I V I E N D A-$ ECONOMICA-TIPO-FENIX-SRR-01---COSTOS--

20. INEC. (2010). Resultados censo 2010. Retrieved April 23, 2018, from http://www.ecuadorencifras.gob.ec/resultados/

21. Ministerio de Ambiente Vivienda y Desarrollo Territorial, C. REGLAMENTO COLOMBIANO DE CONSTRUCCIÓN SISMO RESISTENTE (2010). Colombia.

22. Ministerio de Desarrollo Urbano y de Vivienda, E. Acuerdo Ministerial $\mathrm{N}^{\circ}$ 220 (2011). Ecuador. Retrieved from https://www.habitatyvivienda.gob.ec/wp-

content/uploads/downloads/2013/11/acuerdo_ministerial_no._220_-_27NOV-2013.pdf

23. Ministerio de Desarrollo Urbano y de Vivienda, E. Cargas (no sísmicas) (2015). Ecuador.

24. Ministerio de Desarrollo Urbano y de Vivienda, E. Peligro Sísmico: Diseño Sismo Resistente, Peligro Sísmico: Diseño Sismo Resistente § (2015). Ecuador. Retrieved from http://www.indeci.gob.pe/proyecto58530/objetos/archivos/2011060610284 1.pdf

25. Ministerio de Desarrollo Urbano y de Vivienda, E. Lineamientos mínimos para registro y validación de tipologías de vivienda. (2018). Ecuador. Retrieved from https://www.habitatyvivienda.gob.ec/wpcontent/uploads/downloads/2018/04/LINEAMIENTOS-MINIMOS-PARAREGISTRO-Y-VALIDACION-DE-TIPOLOGIAS-DE-VIVIENDA.pdf

26. Modular Building Institute. (2018). Modular Building Institute. Retrieved May 30, 2018, from http://www.modular.org/HtmlPage.aspx?name=why_modular

27. Modular Home. (2016). Cédula de habitabilidad. Retrieved April 24, 2018, from https://www.modularhome.es/cedula-dehabitabilidad/\#El concepto de habitabilidad

28. Municipalidad de Cuenca, C. Ordenanza plan de ordenamiento territorial (2002). Ecuador.

29. Nieto Cardenas, J. (2014). Diseño de una vivienda de dos plantas con soluciones prefabricadas. Universidad de Cuenca. Retrieved from http://dspace.ucuenca.edu.ec/handle/

30. Panelais Producciones. (2016). Construcción modular. Retrieved April 24, 2018, from http://www.panelais.com/proyectos/construccion-Modular

31. Rivas, R. R. (2007). Ergonomía en el diseño y la producción industrial. Nobuko.

Retrieved

from https://books.google.es/books?hl=es\&lr=\&id=QBoGOgb2b5cC\&oi=fnd\&pg $=P A 9 \& d q=$ producción+industrial+definición\&ots=xge0aP-

$\mathrm{dOL \& sig=TLQDttQ41UpKQ7iWeAmjvMCGjXE \# v=onepage \& q=producción}$ industrial definición\&f=false

32. Sistemas de Información Ejecutiva. (2013). Sistemas Constructivos. Apunte $N^{0}: 1$-Conceptos Básicos, 3. Retrieved from http://www.eis.unl.edu.ar/z/adjuntos/446/Apunte_N_1.pdf

33. Terrados-Cepeda, F. J., Baco-Castro, L., Moreno-Rangel, D., \& MorenoRangel, D. (2015). Vivienda prefabricada, sostenible, autosuficiente y energéticamente eficiente. Participación en la competición Solar Decathlon Europe 2012. Informes de La Construcción, 67(538), e088. 
https://doi.org/10.3989/ic.13.138

34. Vizuete Víctor. (2014, August 12). La construcción informal es la de mayor vulnerabilidad.

Retrieved

from

http://www.elcomercio.com/actualidad/construccion-informal-

vulnerabilidad-sismos-quito.html 